\title{
Infective endocarditis associated with acute renal failure: Repeat renal biopsy and successful recovery
}

\author{
AIFENG WANG $^{2}$, YONGPING WANG ${ }^{1,2}$, GUOBAO WANG $^{1}$, ZHANMEI ZHOU $^{1}$ and XIAOBING YANG ${ }^{1}$ \\ ${ }^{1}$ Division of Nephrology, Nanfang Hospital, and ${ }^{2}$ Department of Forensic Medicine, \\ Southern Medical University, Guangzhou 510515, P.R. China
}

Received December 7, 2009; Accepted February 19, 2010

DOI: 10.3892/etm_00000067

\begin{abstract}
Infective endocarditis (IE) is a serious disease with a high associated mortality rate, particularly when complicated by acute renal failure (ARF). Although valve surgery and treatment with antibiotic agents are recommended, surgical options and the optimal therapy are not as yet well documented. Here, we report a rare case of IE in a young man with a history of intravenous drug abuse, who presented with high fever, bilateral thoracalgia, lower limb edema and renal dysfunction. After treatment with antibiotics, hemodialysis and anticoagulants, a tricuspid valve replacement surgery was performed on the patient. After surgery, his renal functions deteriorated and progressed to ARF. The first renal biopsy showed type II crescentic glomerulonephritis. After receiving continuous ambulatory peritoneal dialysis followed by administration of an angiotensin converting enzyme inhibitor and angiotensin receptor blockers, the patient's serum creatinine level decreased and the urine output increased gradually. In order to identify the renal turnover, we performed a second biopsy and found significant improvement in the pathological changes with endocapillary proliferative glomerulonephritis and fibrous crescents. Successful recovery of renal function was achieved 12 weeks after the initiation of therapy. Therefore, eradication of infection, tricuspid valve replacement and renal substitution therapy may be sufficient in some cases.
\end{abstract}

\section{Introduction}

Despite improvements in diagnostic methods and antimicrobial agents, the incidence of infective endocarditis (IE) remains high in specific populations of individuals, such as hemodialysis patients

Correspondence to: Dr Yongping Wang, ${ }^{2}$ Present address: Shriners Hospital, Rm. 602, 2425 Stockton Blvd., Sacramento, CA 95817, USA E-mail: wypwang@ucdavis.edu

Professor Guobao Wang, Division of Nephrology, Nanfang Hospital, Southern Medical University, 1838 North Guangzhou Street, Guangzhou 510515, P.R. China

E-mail:wgbandyl@medmail.com.cn

Key words: infective endocarditis, acute renal failure, repeat renal biopsy, recovery and drug abusers $(1,2)$. The prognosis of IE has been shown to be strongly influenced by the complication of congestive heart failure, neurologic events, systemic embolism and prolonged fever (3). Glomerulonephritis, secondary to endocarditis, is a rare diagnosis usually associated with blood culture-positive bacteria, particularly staphylococci and streptococci (4). In some cases, IE induces rapidly progressive glomerulonephritis and results in end-stage renal failure, which is associated with poor patient prognosis (5). Apart from antibiotic therapy, effective strategies include surgery, steroid therapy, immunosuppressive therapy and dialysis $(6,7)$. However, the appropriate therapy for IE associated with renal injury has not been adequately defined. Here, we report a case of IE associated with acute renal failure (ARF) for which the clinical symptoms were successfully improved by treatment with antibiotics, surgery and continuous ambulatory peritoneal dialysis (CAPD) therapy. Most importantly, we performed repeat biopsy to elucidate the prognosis and turnover of this condition.

\section{Case presentation}

A 25-year-old male with a history of intravenous drug abuse was admitted to our hospital due to high fever, bilateral thoracalgia, lower limb edema and deterioration of renal function [creatinine (CR), $528 \mu \mathrm{mol} / \mathrm{l}$; blood urea nitrogen (BUN), $12.2 \mathrm{mmol} / \mathrm{l}]$. There was no history of skin rashes, joint swelling, digestive symptoms, respiratory symptoms or hypertension.

One month before admission, the patient was admitted elsewhere with high fever, shivers, general malaise, bilateral thoracalgia and a squeezing feeling. Laboratory tests revealed $798.7 \mu \mathrm{mol} / 1$ of serum CR, $23.4 \mathrm{mmol} / \mathrm{l}$ of BUN and $15.08 \times 10^{9} / 1$ of white blood cells with $73.9 \%$ neutrophilic granulocytes. The blood specimen for culture demonstrated Staphylococcus aureus. His chest CT showed hematogenous dissemination of pulmonary abscesses, and his echocardiogram demonstrated vegetation on the tricuspid valve associated with severe regurgitation. The sizes of the left atrium, right atrium and right ventricle were mildly increased. The patient was treated with antibiotics, hemodialysis and anticoagulation therapy.

After being transferred to our hospital for further treatment, his condition was reevaluated. His temperature was $37^{\circ} \mathrm{C}$, pulse and respiratory rates were 88 and $20 / \mathrm{min}$, respectively, and blood pressure was $105 / 65 \mathrm{mmHg}$. From his physical examination, the patient was diagnosed with an expanded 


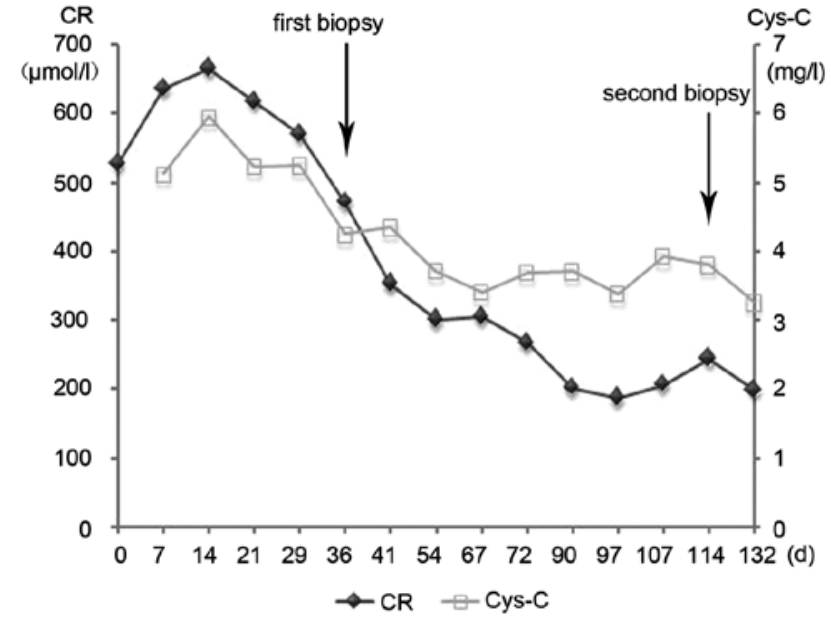

Figure 1. Clinical course of the renal dysfunction in the infective endocarditis patient. The left and right vertical axes represent the levels of serum creatinine $(\mathrm{CR})$ and cystatin $\mathrm{C}(\mathrm{Cys}-\mathrm{C})$, respectively.

heart boundary, weak heart beat and systolic murmur of Levine II/IV. A systolic murmur was noted at the auscultation area of the tricuspid and the second auscultation area of the aortic. The lungs were clear, and the abdomen was soft with no distension, tenderness or palpable masses. The liver was palpable in the left subcostal region and was not tender. The rest of the physical examination was normal. Our overall laboratory findings were anemia ( $\mathrm{Hb}, 73 \mathrm{~g} / \mathrm{l}$; HCT, 0.234); white blood cells, 10.61x10\%/1 (neutrophilic granulocytes 63.1\%); BUN, $12.2 \mathrm{mmol} / \mathrm{l}$ and CR, $528 \mu \mathrm{mol} / \mathrm{l}$. The patient was positive for the hepatitis $\mathrm{C}$ antibody (HCV-Ab). Other parameters, such as serum complement levels, HIV, hepatitis B surface antigen, antinuclear antibody, antiglomerular basement membrane antibody and anti-neutrophil cytoplasmic antibody were all negative or normal. Echocardiogram again showed vegetation on the tricuspid valve with severe regurgitation and expansion of the right atrium and ventricle boundary, accom- panying mild pulmonary hypertension and mild regurgitation of the bicuspid and pulmonary valves.

After the short-term treatment with continuous antibiotics (vancomycin) and hemodialysis, the patient's fever and edema were reduced. However, his renal function did not return to normal (Fig. 1). In the absence of any obvious contraindication to surgery, the patient underwent a successful tricuspid valve replacement, and the treatment with antibiotics, hemodialysis and anticoagulation was continued. There were no bacteria growths in the cultured vegetation and blood specimens. However, the renal function continued to deteriorate, and serum CR increased to $640 \mu \mathrm{mol} / 1$. The patient was then transferred to the Division of Nephrology for treatment of renal insufficiency. A kidney biopsy was performed 6 days later which showed typical crescentic glomerulonephritis (Fig. 2a-c). Eleven of thirteen glomeruli had crescent formation, including eight large fibrocellular crescents, one large fibrous crescent, one small cellular crescent and one small fibrocellular crescent. From the PAS and PASM stainings, an overall interstitial inflammatory cell infiltration, mild tubular atrophy and interstitial fibrosis were observed (Fig. 2). Immunofluorescence microscopy showed the deposition of immune complexes in the glomeruli. Mesangial regions and glomerular capillary walls had comma-like IgA and C3 stainings (1+) diffusely and globally (data not shown).

Considering his severe renal injury and chronic hepatitis $\mathrm{C}$ development, we decided to perform long-term renal replacement therapy and decrease his proteinuric level with a full dose of angiotensin converting enzyme inhibitor (ACEI) and angiotensin receptor blockers (ARBs), rather than immunosuppressive therapy. Since the patient was a drug abuser, he received CAPD with the ultrafiltration volume of $800 \mathrm{ml} /$ day instead of an arteriovenous fistula operation. One month later, he developed profound edema of the right lower extremity and a thrill was palpated in his right groin. Angiography showed right deep femoral arteriovenous fistula because of his repeated puncture. After blocking his right deep femoral arteriovenous fistula using digital subtraction angiography

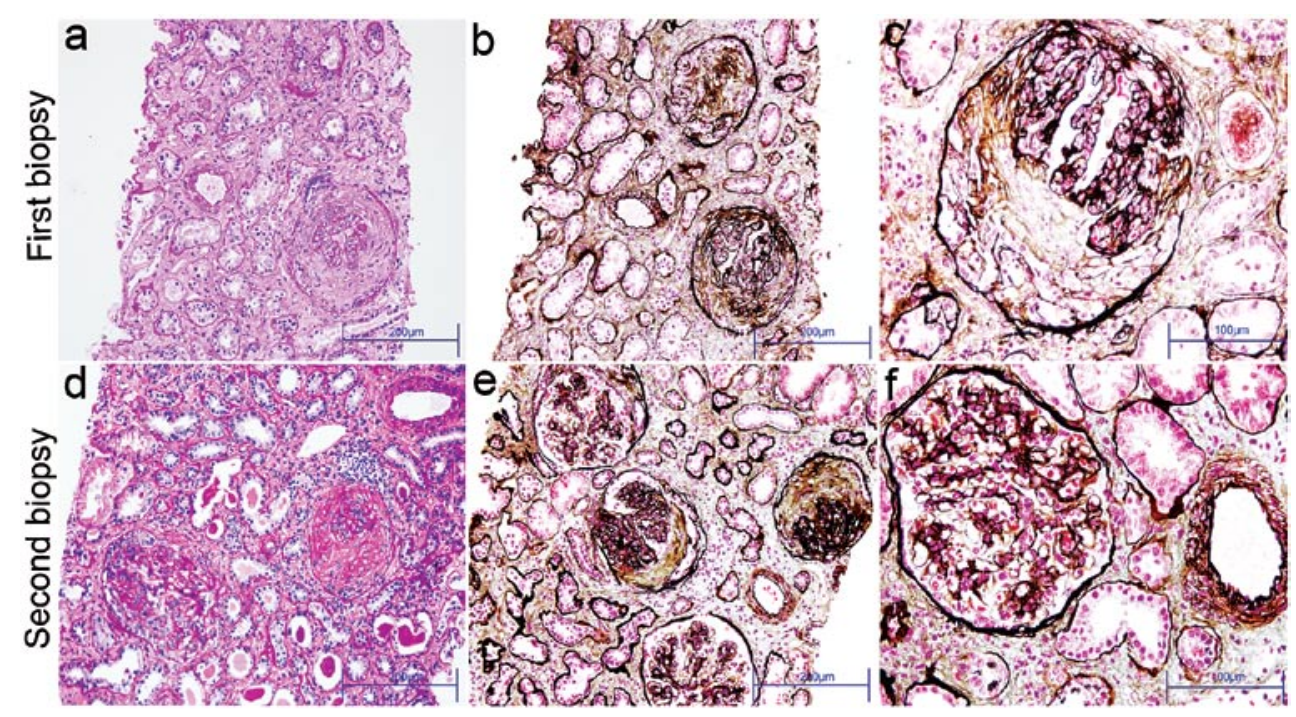

Figure 2. Pathological changes of the infective endocarditis-associated glomerulonephritis in the first and repeat renal biopsy specimens. The first biopsy (a-c) shows typical crescentic glomerulonephritis. The second biopsy (d-f) presents endocapillary proliferative glomerulonephritis with crescent formation (a and d, PAS stain; b, c, e and f, PASM counterstain Trichrome stain; scale bar, $100 \mu \mathrm{m}$ ). 
(DSA), the edema of the right lower leg disappeared. After a 40-day therapy, with a greatly improved condition of a daily urine output of 1,500 ml and serum CR of 180-240 $\mu \mathrm{mol} / \mathrm{l}$, CAPD was terminated. We carried out a repeat biopsy to identify his prognosis and turnover. The second biopsy revealed significant reduction in glomeruli crescents (9/18). They were mostly fibrous components that consisted of five large and two small fibrous crescents, and two small fibrocellular crescents. Proliferation of mesangial and endothelial cells was approximately the same as in the first biopsy. There was little improvement of tubular atrophy, interstitial fibrosis and inflammatory cell infiltration (Fig. 2d-f). Immunoflourescence examination of five glomeruli revealed $2+$ granular IgM and C3 in a global mesangial and capillary wall distribution, and CIq 1+ granular in a segmental mesangial distribution (data not shown). After carefully comparing the pathological changes with the previous biopsy, we diagnosed endocapillary proliferative glomerulonephritis with crescent formation.

\section{Discussion}

Diffuse crescent formation in IE with renal involvement is rare. There is a universal consensus among clinical physicians concerning the treatment of these types of symptoms using antimicrobial therapy $(3,5)$, yet there has been no definitive agreement on the use of other treatments such as surgery and steroid therapy $(1,8,9)$. Furthermore, plasmapheresis treatment is also recommended and is thought to possibly relieve immune-mediated pathogenesis $(10,11)$. Here, we presented a patient who developed crescentic glomerulonephritis accompanying bacterial endocarditis caused by Staphylococcus aureus. Although Staphylococcus aureus is considered the most common pathogen in IE, the mortality rates of chronic hemodialysis patients are quite high when they are infected with methicillin-resistant staphylococcus aureus (MRSA) $(1,12)$. In our patient, IE was diagnosed on the basis of the clinical presentation, positive blood cultures and transesophageal echocardiogram findings. There have been reports of predominant $\operatorname{IgA}$ deposits in glomerulonephritis associated with MRSA infections $(13,14)$. In our case, immunocomplex deposits (IgA, C3, IgM and C1q) were found in the glomerulus of both biopsies, which may be one of the main pathogenic factors for IE. Since the pathogen for this patient was sensitive to vancomycin, his high fever and pulmonary symptoms were dramatically relieved after therapy with antibiotics. However, the antibiotic therapy alone was only able to suppress circulating bacteremia and failed to decrease the size of the vegetation and the nest of bacteria. We decided that it was necessary to perform a tricuspid valve replacement in order to eliminate the vegetation and focal infection. However, his renal function deteriorated a month after surgery. The first renal biopsy was perfomed, revealing extensive crescents in $85 \%$ of the glomeruli. The infection-related circulating immune complex-mediated glomerulonephritis seemed to be the most appropriate mechanism for the deterioration of the renal function (15).

Some case reports have shown that immunosuppressive therapies such as cyclophosphamide and steroid therapy (low-dose) with antibiotics improve renal dysfunction of IE-induced crescentic glomerulonephritis $(6,8)$. Due to the patient's severe renal injury and hepatitis $\mathrm{C}$ infection, we concluded that using immunosuppressive therapy would have a high virus extension risk, and that using renal replacement therapy would be relatively safe. At the same time, there were no relevant reports in the literature clarifying the side effects associated with steroid therapy in this condition. We felt more optimistic when his renal dysfunction obviously improved after the patient accepted CAPD and was administered a full dose of ACEI and ARBs. More importantly, we performed a repeat biopsy and found better turnover of the pathological changes. The proportion of crescents in the yielded glomeruli was less than that of the first biopsy and mainly consisted of a fibrous component. Based on the second biopsy, we verified a diagnosis of endocapillary proliferative glomerulonephritis with crescent formation. As mentioned above, the percentage of crescents was 11/13 (85\%) in the first biopsy and 9/18 (50\%) in the second. Notably, the decrease in the proportion of crescents from the first to the second biopsy was modified by the inclusion of the absence of statistical significance and the possibility of sampling variation. After treatments to eliminate the focal infection, decrease the proteinuria and protect the renal function, the patient recovered to a urine output of $1,000-2,000 \mathrm{ml} / 24 \mathrm{~h}$ and a serum CR of $120-200 \mu \mathrm{mol} / 1$.

The patient prognosis of IE associated with extensive crescents is poor, and patients usually present with ARF (16). In the event of a delayed diagnosis and inappropriate antibiotic therapy, the condition of the patient may deteriorate leading to death. After effective antibiotic and surgical therapy, continual protection and recover of the renal function is the principal objective. Our previous research revealed that ACEI therapy significantly reduces the level of proteinuria and delays the rate of decline in renal function (17). However, patients with IE-associated glomerulonephritis were not included in this report. Here, we used an adequate dosage of ACEI and ARBs in order to decrease the patient's proteinuria, and his renal function was successfully recovered with CAPD therapy. The pathophysiology and prognosis of this case are perhaps similar to those of postinfectious glomerulonephritis, which involves exposure to bacterial antigens and can sometimes be crescentic. In conclusion, the present report describes the clinical presentation, histological outcome and effective treatment of a patient with IE and crescentic glomerulonephritis caused by Staphylococcus aureus. Patients with IE associated with ARF can be successfully treated with the proper use of antibiotics, surgery, renal replacement and ACEI/ARB therapies. This presents the clinical and biological implications of kidney protection and proteinuria reduction in the treatment of this illness.

\section{Acknowledgements}

We thank Kai Wang (University of California, Davis) for editing the manuscript.

\section{References}

1. Spies C, Madison JR and Schatz IJ: Infective endocarditis in patients with end-stage renal disease: clinical presentation and outcome. Arch Intern Med 164: 71-77, 2004.

2. Levine DP, Cushing RD, Jui J, et al: Community-acquired methicillin-resistant Staphylococcus aureus endocarditis in the Detroit Medical Center. Ann Intern Med 97: 330-338, 1982. 
3. Mylonakis E and Calderwood SB: Infective endocarditis in adults. N Engl J Med 345: 1318-1330, 2001.

4. Majumdar A, Chowdhary S, Ferreira MAS, et al: Renal pathologic findings in infective endocarditis. Nephrol Dial Transplant 15: $1782-1787,2000$

5. Neugarten J, Gallo GR and Baldwin DS: Glomerulonephritis in bacterial endocarditis. Am J Kidney Dis 3: 371-379, 1984.

6. Rovzar MA, Logan JL, Ogden DA, et al: Immunosuppressive therapy and plasmapheresis in rapidly progressive glomerulonephritis associated with bacterial endocarditis. Am J Kidney Dis 7: 428-433, 1986.

7. Kannan S and Mattoo TK: Diffuse crescentic glomerulonephritis in bacterial endocarditis. Pediatr Nephrol 16: 423-428, 2001.

8. Koya D, Shibuya K, Kikkawa R, et al: Successful recovery of infective endocarditis-induced rapidly progressive glomerulonephritis by steroid therapy combined with antibiotics: a case report. BMC Nephrol 5: 18-22, 2004.

9. Rankin JS, Milford-Beland S, O'Brien SM, et al: The risk of valve surgery for endocarditis in patients with dialysis-dependent renal failure. J Heart Valve Dis 16: 617-622, 2007.

10. Daimon S, Mizuno Y, Fujii S, et al: Infective endocarditisinduced crescentic glomerulonephritis dramatically improved by plasmapheresis. Am J Kidney Dis 32: 309-313, 1998.
11. Couzi L, Morel D, Deminière C, et al: An unusual endocarditisinduced crescentic glomerulonephritis treated by plasmapheresis Clin Nephrol 62: 461-464, 2004

12. Nori US, Manoharan A, Thornby JI, et al: Mortality risk factors in chronic haemodialysis patients with infective endocarditis Nephrol Dial Transplant 21: 2184-2190, 2006.

13. Koyama A, Sharmin S, Sakurai H, et al: Staphylococcus aureus cell envelope antigen is a new candidate for the induction of $\operatorname{Ig} \mathrm{A}$ nephropathy. Kidney Int 66: 121-132, 2004.

14. Koyama A, Kobayashi M, Yamaguchi N, et al: Glomerulonephritis associated with MRSA infection: a possible role of bacterial superantigen. Kidney Int 47: 207-216, 1995.

15. Rames L, Wise B, Goodman JR, et al: Renal disease with Staphylococcus albus bacteremia. A complication in ventriculoatrial shunts. JAMA 212: 1671-1677, 1970.

16. Lerner IL and Weinstein L: Infective endocarditis in the antibiotic era. N Engl J Med 274: 199-206; 259-266; 320-321, 1966.

17. Hou FF, Zhang X, Zhang GH, et al: Efficacy and safety of benazepril for advanced chronic renal insufficiency. N Engl J Med 354: 131-140, 2006. 